\title{
La situación del turismo comunitario en Ecuador
}

\section{The situation of community tourism in Ecuador \\ A situação do turismo comunitário no Equador}

\author{
Carlos A. Vargas-Cumbajín ${ }^{I}$ \\ jmendez@uce.edu.ec \\ Sebastián G. Yánez-Segovia II \\ sgyanez@uce.edu.ec \\ Herman W. Hernández-Benalcázar III \\ hhernandez@uce.edu.ec
}

\author{
Jhony F. Méndez-Játiva IV \\ jmendez@uce.edu.ec \\ Washington R. Valdiviezo-Leroux V \\ rvaldiviezoleroux@yahoo.com \\ Valdano Tafur VI \\ vtafur@uce.edu.ec
}

Recibido: 20 de octubre de 2017 * Corregido: 20 de noviembre de 2017 * Aceptado: 01 enero de 2018

I. Biólogo, Docente de la Universidad Central del Ecuador, Quito, Ecuador.

II. Ingeniero Agrónomo, Docente de la Universidad Central del Ecuador, Quito, Ecuador.

III. Biólogo, Docente de la Universidad Central del Ecuador, Quito, Ecuador.

IV. Ingeniero Agroindustrial, Docente de la Universidad Central del Ecuador, Quito, Ecuador.

V. Economista, Docente de la Universidad Central del Ecuador, Quito, Ecuador.

VI. Ingeniero Agrónomo, Docente de la Universidad Central del Ecuador, Quito, Ecuador. 


\section{Resumen}

Este estudio se realizó en 36 Comunidades y/o Centros de Turismo Comunitario (CTC) del Ecuador, el mismo que da cuenta sobre el impacto de esta actividad en el ámbito social, ambiental y de sostenibilidad de los emprendimientos relacionados con el sector, donde se encuentra un segmento de la población más vulnerable de la sociedad ecuatoriana, como son los habitantes de las áreas rurales. También se analizó la situación real del turismo y su influencia social, económica y ambiental como rama de la actividad dedicada a la prestación de los servicios y atención a los visitantes que buscan conseguir la satisfacción de las necesidades espirituales, de conocimiento, recreación, descanso personal y familiar. El estudio evidencia los aportes positivos del Turismo Comunitario en Ecuador, como son la generación de puestos de trabajo, el posicionamiento en mercados no tradicionales, la utilización racional de los recursos naturales, bases fundamentales del turismo ecológico y la responsabilidad social - comunitaria a partir de la organización comunitaria, la identificación de los recursos disponibles para el turismo; así como se evidencian algunas debilidades en cuanto a la disponibilidad de la infraestructura básica, promoción y la deficiente organización laboral en el sector público.

Palabras clave: Servicios turísticos, cultura, estructura organizacional. 


\section{Abstract}

This study was carried out in 36 Communities and / or Community Tourism Centers (CTC) of Ecuador, which reports on the impact of this activity in the social, environmental and sustainability aspects of the projects related to the sector, where it finds a segment of the most vulnerable population of Ecuadorian society, such as the inhabitants of rural areas. We also analyzed the real situation of tourism and its social, economic and environmental influence as a branch of activity dedicated to the provision of services and attention to visitors seeking to achieve the satisfaction of spiritual needs, knowledge, recreation, personal rest and family The study evidences the positive contributions of Community Tourism in Ecuador, such as the generation of jobs, the positioning in non-traditional markets, the rational use of natural resources, fundamental bases of ecological tourism and social-community responsibility from the community organization, the identification of the resources available for tourism; as well as some weaknesses in terms of the availability of basic infrastructure, promotion and poor labor organization in the public sector.

Key words: Tourist services, culture, organizational structure. 


\section{Introducción.}

Preliminarmente al desarrollo del Neoliberalismo, el turismo se fue vendiendo como una estrategia fácil y rápida de desarrollo y lucha contra la pobreza, capaz de generar elevados beneficios con muy poca inversión, ya que su principal elemento de atracción (el paisaje y la cultura autóctona) ya estaban gratuitamente establecidas. (Burns \& Holden, 1995; Mowforth \& Munt, 1998; Hamilton, 2006; Hickman, 2007; Nowicka, 2008) Este discurso, encabezado por la Organización Mundial del Turismo y legitimado cuando esta institución multilateral entró en la estructura de Naciones Unidas como agencia especializada a principios de la década del 2000. (Duterme, 2006), fue calando con éxito en todos los estratos de la sociedad latinoamericana. (Mowforth, Charlton, \& Munt, 2008)

Los modelos de turismo dominantes, como el de enclave, que se han mostrado muy agresivos con el mundo rural (Feeroz, 2009), algunas experiencias parecían sugerir que era posible apostar por un turismo gestionado por unidades familiares campesinas, por comunidades, por cooperativas o por pueblos indígenas, integrando este tipo de actividad de forma complementaria a las tradicionales (Ruiz-Ballesteros, 2011). Una propuesta que incluso podía ser coherente con un modelo de desarrollo rural basado en una estrategia de Soberanía Alimentaria (Gascon \& Cañada, 2005). El turismo así gestionado, y al que en las últimas décadas se ha venido a denominar turismo rural comunitario, agroturismo o turismo campesino entre otras denominaciones, debía ayudar a la consolidación de las maltrechas economías campesinas latinoamericanas ofreciendo un aporte de recursos económicos y diversificando sus fuentes de ingresos. Además, el turismo rural comunitario coincidía perfectamente con las tendencias post-fordistas crecientes en el turismo internacional. 
Entendemos por turismo rural comunitario un tipo de turismo de pequeño formato, establecido en zonas rurales y en el que la población local, a través de sus estructuras organizativas, ejerce un papel significativo en su control y gestión. Esta definición es muy laxa, pero permite englobar toda la variabilidad de experiencias existentes (Gascon \& Cañada, 2005). Igualmente, el turismo rural comunitario es destacable porque se dirige a los sectores más desfavorecidos de la sociedad y establece sinergias con otras políticas de desarrollo (económicas en el ámbito agropecuario, de patrimonio cultural, medioambientales).

Otro ámbito de debate es su impacto en las relaciones de género: si bien a menudo se plantea que puede tener efectos positivos, tales como la mejora de la autoestima de la mujer, también se descubre que puede acabar consolidando sus roles tradicionales y que asume la mayor parte del esfuerzo de la nueva actividad, ya que comporta especialmente tareas del ámbito doméstico como el alojamiento o la alimentación de los visitantes (Henrici, 2007). Diversos estudios plantean el riesgo de que las actividades tradicionales se degraden por una desviación de la fuerza de trabajo disponible hacia la nueva actividad. También se ha detectado la dificultad de la población local para controlar y gestionar una actividad compleja y para la que tienen escasa o nula formación (Sasha Davis \& Morais, 2004), o que mayoritariamente son iniciativas promovidas por agentes foráneos (ONG, políticas gubernamentales) y no propuestas endógenas (Paniagua, 2002). Igualmente se le acusa de ser un mecanismo a nivel "micro" de mercantilización de la naturaleza (Duffy, 2008). Incluso se empieza a dudar de su real capacidad para generar ingresos que permitan reducir los niveles de pobreza de la población local, factor que era uno de sus principales atractivos. (Deller, 2010) 
Hacia fines de los años 1950 en Ecuador, en el marco de políticas modernizantes promovidas por el Gobierno de Galo Plaza, se propone al turismo internacional como un instrumento para el desarrollo del país para lo cual se establece una oficina adscrita a la Presidencia del República. En este sentido, la llamada "misión cultural indígena", presidida por Rosa Lema, marca un hito relevante orientado a publicitar el país pero especialmente a la zona de Otavalo, en el mercado norteamericano (Prieto, 2008). Las poblaciones locales han sido incluidas en la generación de espacios turísticos, bajo criterios de inclusión y de sostenibilidad ecológica (Grab \& Linde, 2008); y, también en la búsqueda de nichos de mercado que atienden las demandas del "nuevo turismo", que muestra su preferencia por "formas de ecoturismo, turismo mochilero y el viaje independiente" que suele ser "postfordista" y de "pequeña escala" (Hampton, 2003). El nuevo turismo, a diferencia del turismo masivo de grandes capitales, no solamente representa una demanda del mercado sino también una estrategia desde sectores subalternos para diversificar la producción. En el Ecuador esta estrategia ha surgido, entre muchas otras, tras varias décadas en las cuales tanto el Estado como las organizaciones de base han buscado modelos de desarrollo rural que afronten la inmensa y persistente desigualdad socio-económica entre la ciudad y el campo. Hasta los años 1980, los grandes proyectos rurales tales como el Programa Nacional de Desarrollo Rural (PRONADER) y los proyectos del Fondo Ecuatoriano Populorum Progressio (FEPP) no habían logrado los resultados esperados. Han sido criticados por sus "enfoques sectorialistas, proyectistas y productivistas" (Martínez, 2003) y hasta por ser "irrelevantes" en términos de reparar desigualdades económicas dentro de un contexto macroeconómico neoliberal (North \& Cameron, 2003). Para finales de la década de los años 1990, se acumulaban varias críticas al desarrollo económico en general como un discurso hegemónico de dominio etnocéntrico o imperialista tanto desde la academia como desde organizaciones de base, mientras ellos proponían modelos de cambio que fortalecieran comunidades 
vulnerables sin reestructurar sus economías, imponer lógicas culturales ajenas ni forzar su integración en el mercado global.

El involucramiento comunitario dio a la luz su propia cosmovisión partiendo del concepto real donde "Es la relación de la comunidad con los visitantes desde una perspectiva intercultural, en el contexto de los viajes organizados, el manejo adecuado de los recursos naturales, la valoración de sus patrimonios, los derechos culturales y territoriales de las nacionalidades y pueblos para la distribución equitativa de los beneficios generados". Ecuador es el país líder en Turismo Comunitario, depositario de una rica experiencia en emprendimientos turísticos que son el resultado de un debate consensuado entre los miembros de distintas nacionalidades, pueblos, culturas y diversidad étnica desde una iniciativa endógena de las comunidades bajo orientaciones de sus líderes que posteriormente se materializan gremialmente en la Federación Plurinacional de Turismo Comunitario del Ecuador (FEPTCE). (Hutchins, 2007)

La tendencia de la demanda turística por las diferentes modalidades es incremental, desde la perspectiva del: agroturismo, turismo de aventura, ecoturismo, turismo vivencial; los más solicitados, especialmente si son gestionadas por las comunidades locales que transmiten su sabiduría ancestral en cada paso de las actividades de prestación de los servicios turísticos; sin embargo, son pocos los trabajos relacionados con el impacto del turismo en los aspectos: ambiental, sociocultural y económico, tampoco existe un seguimiento de la sostenibilidad de los emprendimientos en recursos naturales, culturales, infraestructuras y niveles de participación comunitaria. Es importante visibilizar los vacíos que contribuirán en la toma de decisiones y orientaciones en el turismo bajo gestión comunitaria que reflejen información real de las fortalezas y 
debilidades existentes en el sistema comunitario. En el Ecuador no existen estudios concretos sobre el impacto del turismo comunitario y solamente hay estudios temáticos en comunidades específicas.

\section{Metodología.}

Se la realizó en dos instancias, una primera de recopilación de la información bibliográfica y la segunda el trabajo de campo de cara a los gestores donde la observación y la entrevista a los informantes. Se contempló un diseño metodológico para el análisis de las experiencias de turismo comunitario en Ecuador. Se seleccionaron las técnicas e instrumentos a aplicarse al grupo focal de estudio en las dimensiones de sostenibilidad económica, social, cultural y ambiental. Las encuestas fueron aplicadas por estudiantes de la Cátedra de Turismo Comunitario en 43 comunidades y/o centros de turismo comunitario, en once provincias de Ecuador. La información fue sistematizada en el programa Excel y elaborados los cuadros estadísticos, para finalmente ser analizados e interpretados. Siete de las encuestas fueron eliminadas por debilidades en la información existente en esas comunidades, total 36 encuestas válidas que equivalen al 100\%. Se validaron, para finalmente aplicarlos en las comunidades seleccionadas (Tabla 1). Las comunidades fueron escogidas al azar sin tomar en cuenta de si están o no registradas legalmente ante la autoridad competente, es decir, si pertenecen a alguna red u organización; las preguntas fueron seleccionadas en base a los objetivos planteados en cinco ámbitos: cultural, ambiental, infraestructura, económico y turístico. 
Tabla 1. Ubicación y número de las comunidades y/o Centros de Turismo Comunitarios (CTC) en las 11 provincias en estudio del Ecuador Continental.

\begin{tabular}{|lll|}
\hline PROVINCIA & \multicolumn{1}{c}{ COMUNIDAD y/o CTC } & N $^{\circ}$ \\
\hline Azuay & $\begin{array}{l}\text { Huertas, Parcoloma, Principal, Chilca Totoras, Inmaculada } \\
\text { Barabón, Kushi Waira }\end{array}$ & 6 \\
\hline Bolívar & Salinas de Guaranda & 1 \\
\hline Chimborazo & $\begin{array}{l}\text { Guargualla, Balda Lupaxi Bajo, Palacio Real, Calshi, Pulinguí } \\
\text { San Pablo }\end{array}$ & 5 \\
\hline Esmeraldas & Olmedo & 1 \\
\hline Imbabura & Sumak Pacha, Nangulví, Turuco, Mascarilla, Santa Bárbara, & 11 \\
& Carpuela, Cielo Verde -Manduriacos, Tunibamba, La Calera, & \\
\hline Loja & Junín, San Clemente & 3 \\
\hline Manabí & Las Lagunas & 2 \\
\hline Napo & Salango, Casas Viejas, El Pital & 1 \\
\hline Orellana & Sinchi Warmi, Rio Blanco & 4 \\
\hline Pastaza & Sacha Nampy & 1 \\
\hline Tungurahua & Arajuno, Shiwa Kucha, San José Wapuno, CEPLOA \\
\hline
\end{tabular}

\section{Resultados y discusión.}

Ecuador es un país y a la vez un destino privilegiado al contar con muchas ventajas para el turismo; geográficamente se encuentra en la costa noroccidental de América del Sur, atravesado por la Línea Ecuatorial, la Cordillera de los Andes, corrientes marinas, áreas selváticas amazónica y del Chocó Andino, elementos naturales que le hacen un país con ecosistemas cercanos único.

Ecuador se encuentra dentro de los 14 países más biodiversos del mundo con 17 pueblos y nacionalidades indígenas distribuidas en las cuatro regiones naturales. En el presente estudio, estos atributos únicos de la expresión cultural hacen de los CTC una de las características de mayor fortaleza en la región Sierra con el 70\% donde más se ha desarrollado; la Amazonía con el 20\% y 10\% en la región costa (Figura 1). 


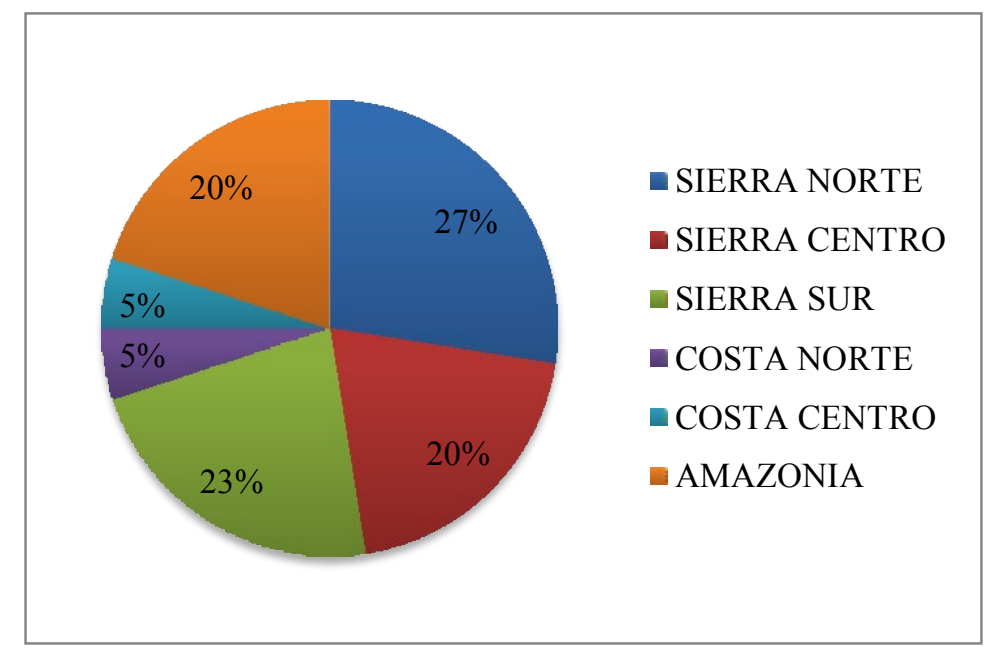

Figura 1. Porcentaje ponderado de ocupación de las comunidades y/o CTC en las regiones del

\section{Ecuador}

Uno de los problemas del turismo comunitario es la deficiente investigación a nivel nacional que permitan disponer de cifras confiables y actualizadas para la toma de decisiones. Tal es el caso de comprobación sobre esta afirmación que la misma FEPTCE, maneja datos estimativos e información cualitativa (Roux, 2013). Desde una aproximación teórica se afirma que el Turismo Comunitario mejora las condiciones de vida de la población, dinamiza la economía local, genera empleos, afianza la conservación ambiental y la defensa de los territorios.

En Ecuador, el turismo tiene bien marcados las temporadas durante el año, así las temporadas altas se registran los meses de (julio, agosto, diciembre) y muy bajas (marzo, abril, septiembre). En Turismo Comunitario, esta estacionalidad constituye una fortaleza ya que se aprovechan las temporadas altas de arribo de visitantes durante el mes de agosto, donde los comuneros se encuentran libres de sus actividades cotidianas (cosechas) y listos para recibir a los visitantes generando una ocupación temporal y/o permanente en la comunidad. Además, el presente estudio permite afirmar que el Turismo Comunitario promueve la equidad de género, al integrar a 
las mujeres en su estructura laboral en un 51\%, dentro de los CTC (Tabla 2); es decir la relación laboral de género cumple la propuesta del programa impulsada por la Organización Naciones Unidas (ONU) Turismo Sostenible y Atenuación de la Pobreza (ST-EP 2002), que tiene relación con el tercer objetivo "ODM-3. Promover la igualdad de género y el empoderamiento de la mujer. (OMT, 2004)

Tabla 2. Porcentaje por ocupación en las comunidades y/o Centros de Turismo Comunitarios (CTC) en las 11 provincias en estudio del Ecuador Continental.

\begin{tabular}{|cccc|}
\hline $\begin{array}{c}\text { TIPO DE } \\
\text { EMPLEO }\end{array}$ & $\mathbf{\%}$ & MUJERES & HOMBRES \\
\hline Permanente & 92 & 51 & 49 \\
\hline Temporales & 98 & 53 & 47 \\
\hline
\end{tabular}

El turismo gestionado por comunidades y pueblos indígenas, afro-ecuatorianas, montubias y mestizos locales constituye un alcance decisivo en el momento de decidir los viajes; los visitantes que optan por esta forma de gestión turística prefieren vivir una experiencia de la cultura local, una forma genuina de hacer turismo que es utilizada por competidores que no tienen estas características y solo utilizan la etiqueta de "comunitario", por lo que es importante conocer el tipo de propiedad del negocio turístico y sus grupos de gestionarías comunitarias. Esta consideración es muy importante para afianzar la gestión comunitaria y según las cifras obtenidas en la presente investigación donde el $53 \%$ es gestionado por la comunidad la misma que reúne las condiciones y exigencias establecidas por la FEPTCE relacionado con el Turismo Comunitario, en este sentido, además las cifras también demuestran que un importante $29 \%$ es gestionado por familias, pero con autorización de la comunidad, el 13\% son emprendimientos privados y un 5\% mixto. 
Es importante considerar que el funcionamiento de los Centros de Turismo Comunitario CTC en Ecuador se encuentra regulado por la Ley y sus reglamentos. “Art. 5.- De la Gestión. - La gestión de la actividad turística corresponde a la comunidad, y como tal se procederá a su Registro en el Ministerio de Turismo, previo al inicio de su funcionamiento" (Reglamento para los Centros Turísticos Comunitarios 2010); al preguntar su condición jurídica en cuanto a su legalización de los CTC, en su mayoría dicen estar legalizados $62 \%$, lo cual es muy positivo, un $33 \%$ dice estar en trámite y un 5\% admite no está legalizado.

\section{Caracterización de los visitantes a los CTC.}

El libro de registro de visitantes en una comunidad representa un documento importante por cuanto permite entender la demanda histórica, el perfil y el índice de satisfacción de los turistas que llegan al CTC; además, el manejo de las estadísticas, la información sobre mercados emisores, datos fundamentales para la planificación de cara al mejoramiento continuo de la calidad del producto turístico.

Las cifras obtenidas en la presente investigación, indican que el 70\% de los visitantes a los centros comunitarios son extranjeros que proceden especialmente de España y Estados Unidos

\section{(Figura 2).}




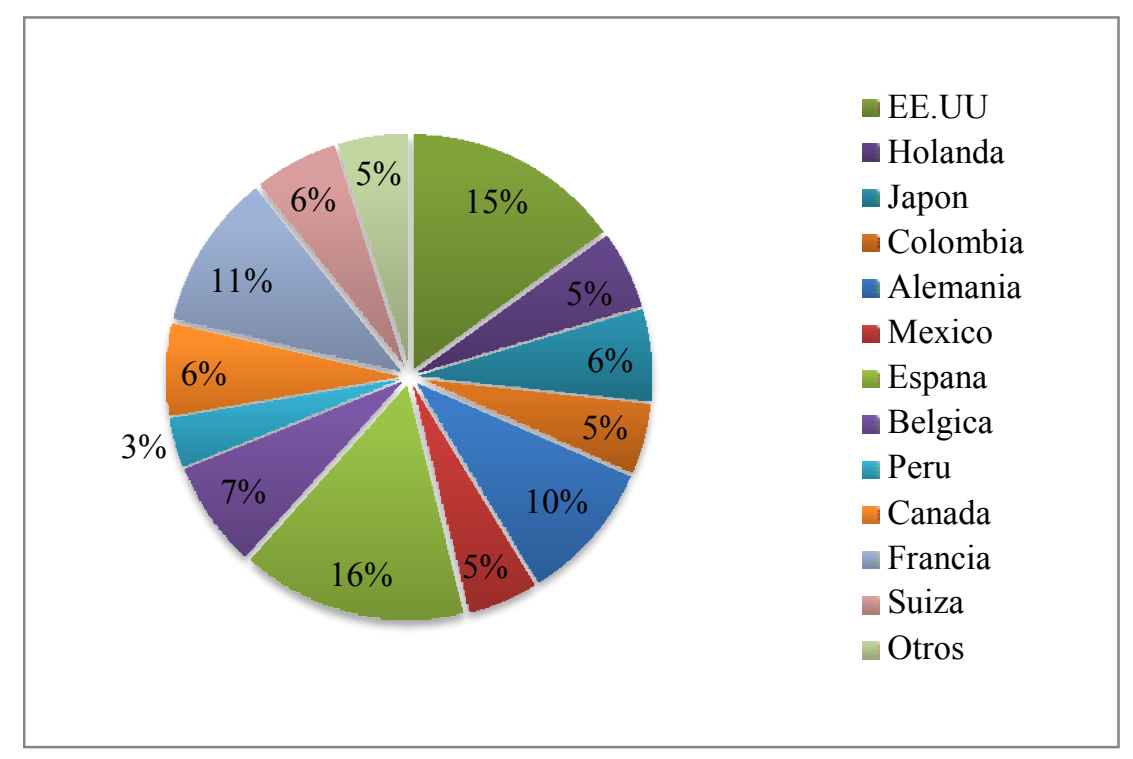

Figura 2. Procedencia de visitantes por país en las comunidades y/o CTC en las regiones del

\section{Ecuador}

Los turistas que visitan los CTC, en su mayoría son viajes familiares por lo que las edades son dispersas pero se ubican entre los de 19 a 60 años, con un 61\%, grupo etéreo interesado en convivir con las comunidades y naturaleza. El código de conducta para visitantes y prestadores de servicios dentro de un CTC es muy importante pues delimita lo permisible y lo no permisible durante la visita, pero tan solo el $74 \%$ de los Centros Comunitarios cuenta con este importante documento y el $26 \%$ no lo tiene.

\section{Servicios Básicos en las comunidades y/o CTC.}

Para el funcionamiento de los CTC y la entrega de un producto de alta satisfacción para el turista es indispensable que cuente con los servicios básicos; el estudio revela una debilidad muy fuerte con relación a este elemento, pues resulta sorprendente que en pleno siglo XXI el 54\% de los 
CTC consumen agua entubada, poniendo en peligro incluso la salud de los visitantes, solo el 23\% tienen acceso a agua potable y un $9 \%$ agua tratada con cloro y filtrada.

El acceso a energía eléctrica para alumbrado en los CTC y comunidades, el $80 \%$ cuentan con acceso al servicio eléctrico público, mientras que los que tienen otras formas de generación de energía como plantas generadoras, paneles solares o generadores con dínamo, son muy pocas. En cuanto a medios de comunicación la tecnología ha llegado a estas comunidades, así el 89\% cuenta con cobertura celular, el $24 \%$ por internet y un $11 \%$ no cuenta con este servicio; en algunas comunidades deben hacer esfuerzos como trasladarse a algún lugar elevado para ubicar la señal y poder comunicarse. El servicio de telefonía móvil en el país es prestado por tres empresas que cubren el servicio de telefonía móvil Claro 47\%, Movistar 32\% y CNT 21\%; también se comunican por otros medios como el teléfono convencional $27 \%$, radio $20 \%$ y el $20 \%$ afirma utilizar correo convencional, entendido como encomiendas por medios de transporte.

En cuanto a servicios de apoyo, como la atención médica, en casos de emergencia la investigación revela que deben acudir a otro poblado más cercano $34 \%$, ya que solo un $24 \%$ cuenta con un centro médico en la comunidad, el $21 \%$ acude a un Shamán o quien realiza limpias y un $13 \%$ cuenta con un botiquín de primeros auxilios que contienen medicamentos básicos.

La seguridad turística es responsabilidad de todos, en los CTC es fundamental, para la comunidad local y los visitantes (Código de Ética Mundial para el Turismo), al respecto los resultados revelan que el $42 \%$ está dado por la policía nacional, el $20 \%$ por los guardias propios de la comunidad, un $20 \%$ manifiesta no contar con seguridad para la comunidad, por lo tanto, para los visitantes y el $9 \%$ cuenta con la ayuda por parte de los guarda-parques en lugares cercanos a las áreas naturales protegidas. 


\section{Gestión de los desechos.}

Los desechos sólidos en las comunidades y CTC vinculadas al turismo, un 35\% entrega al carro recolector, un 19\% quema la basura, el 14\% aprovecha como abono realizando compostaje y el $13 \%$ lo recicla. También es preocupante que solo el $26 \%$ tiene acceso a alcantarillado en los CTC; el resto existe un rudimentario tratamiento de aguas residuales pues el $58 \%$ va a un pozo séptico, un 9\% admite que lo evacúa al río, constituyendo foco de contaminación de los ambientes lacustres y fluviales; ninguna comunidad encuestada posee un programa de tratamiento de aguas residuales.

\section{Redes de Turismo.}

El trabajo coordinado con otras empresas de turismo públicas y privadas cataliza el desarrollo turístico. En el país existen importantes experiencias de asociatividad en redes de turismo gestionadas por comunidades, la mayoría de los CTCs se trabajan en sinergia con otras comunidades y aprovechan de una operadora comunitaria y se benefician de su promoción. Al preguntar si pertenecen o no a una Red de Turismo Comunitario, el $76 \%$ dice sí pertenecer a una red y un 24\% no. En Ecuador la organización más fuerte y reconocida, incluso a nivel internacional es la Federación Plurinacional de Turismo Comunitario del Ecuador (FEPTCE) y resulta que el 64\% pertenecen esta organización. Existen otras redes de Turismo Comunitario reconocidas como la pionera Red Indígena de Comunidades del Alto Napo para la Convivencia Intercultural y el Ecoturismo que abarca a 9 CTC de comunidades Kichwas. 


\section{Servicios Turísticos en los CTC.}

En alojamiento, las comunidades prestan estos servicios, el 32\% en casas de familias miembros de la comunidad, muy común especialmente en la provincia de Imbabura que impulsa la operadora turística RUNATUPARI, mientras que un $28 \%$, se aloja en cabañas, especialmente en CTC de la región Amazónica, el 22\% prefiere acampar y en menor proporción 8\% busca para su pernoctación un hotel cercano. Los tipos de habitación con los que cuenta los CTC para alojamiento son: $37 \%$ sencillas o individuales, el $33 \%$ dobles o matrimoniales, el $24 \%$ son habitaciones triples para familias pequeñas y un $6 \%$ son comunales con camas literas; los administradores dicen que estos alojamientos, un $72 \%$ está en buen estado, un $26 \%$ en estado regular y un $2 \%$ se encuentra en malas condiciones. El baño o servicios higiénicos, se encontró que en el $48 \%$ con baño privado, el 45\% es de acceso compartido y un 7\% todavía utilizan letrinas, especialmente en la región Amazónica.

El servicio de Guías comunitarios, se encontró que el 40\% están capacitados para la guianza, un interesante 27\% tienen Licencia MINTUR y el 33\% son empíricos, algunos mencionan que sus licencias se encuentran caducadas.

\section{El precio de los servicios en los CTC.}

En lo referente a los costos de los servicios de hospedaje, por noche, que ofrecen a los visitantes, se encontró que un $43 \%$ oscila entre $\$ 11-\$ 20$, seguido por un $32 \%$ bajo los $\$ 10$, el $18 \%$ está entre $\$ 21$ y $\$ 40$ y en ninguna comunidad el precio supera los $\$ 81$; estos valores dicen incluir cena, alojamiento y desayuno el 51\%, el 36\% solo la pernoctación y el 13\% que incluye alojamiento y desayuno. 
En cuanto al proceso de comercialización de los paquetes turísticos, algunas comunidades se han preocupado y mantienen convenios con operadoras turísticas nacionales y extranjeras para asegurar la llegada de visitantes, un importante 46\% dijo que sí tenían con operadoras nacionales, el $15 \%$ con operadoras internacionales, pero también un preocupante $39 \%$ dijo que no tenían ningún convenio, constituyendo su mayor debilidad y la razón de que no llegan los visitantes poniendo en peligro el fracaso de los proyectos.

El turismo gestionado por las comunidades locales, constituye una oportunidad para vender de forma directa a los visitantes nacionales, sus productos cosechados, semielaborados o elaborados, especialmente los visitantes nacionales gustan llevar productos de la comunidad, así tenemos que el $51 \%$ adquieren artesanías, el 25\% llevan frutas de temporada de la localidad, el 18\% gusta 1levar inclusive verduras producidas en la localidad, el 4\% llevan mariscos y un $2 \%$ productos reciclados; también compran en las comunidades, productos ya elaborados como: queso y mermelada el $26 \%$, licores el 15\%, yogurt y chocolates el $7 \%$.

Según el MAE (Ministerio del Ambiente del Ecuador, 2008) “... el turismo comunitario representa un paso importante en el concepto de economía turística, ya que permite a las comunidades locales beneficiarse total o mayoritariamente de esta actividad, siendo capaces de manejar la actividad de manera acorde con su propia cultura. Por otro lado, fomenta la conservación de los recursos naturales y culturales, al crearse una conciencia de la importancia de los mismos como atractivos para la actividad turística.

El turismo comunitario es una forma de gestión de diferentes tipos y actividades turísticas que realizan en los CTC con participación directa de las comunidades locales. La riqueza cultural de las comunidades, la diversidad biológica, paisajística, gastronómica, el visitante las disfruta en las 
múltiples actividades que ofrecen y se acoplan a los segmentos por edad, gustos y preferencias de los visitantes, siendo el turismo de naturaleza el más demandado con el disfrute de observación de la flora y fauna, especialmente el aviturismo; en turismo de aventura la actividad preferida es el senderismo; en turismo cultural gusta mucho convivir con las familias por su amabilidad y calidez; mientras que en agroturismo la actividad preferida es participar en siembra y cosecha de los diversos cultivos. Los visitantes buscan calmar sus dolencias recurriendo a medicina ancestral: rituales shamánicos, curaciones y limpias aprovechando al máximo su viaje hacia las comunidades y en turismo histórico visita y admira las culturas en museos, sitios históricos y ruinas arqueológicas (Tabla 3). 
Tabla 3. Principales tipos y actividades turísticas en las comunidades y/o Centros de Turismo

\section{Comunitarios (CTC) en las 11 provincias en estudio del Ecuador Continental.}

\begin{tabular}{|c|c|c|c|}
\hline $\begin{array}{l}\text { TIPO DE } \\
\text { TURISMO }\end{array}$ & $\%$ & $\begin{array}{c}\text { ACTIVIDADES CON } \\
\text { PARTICIPACIÓN COMUNITARIA }\end{array}$ & $\%$ \\
\hline \multirow{3}{*}{$\begin{array}{l}\text { De } \\
\text { Naturaleza }\end{array}$} & \multirow{3}{*}{23} & Observación y fotografía de flora y fauna & 32 \\
\hline & & Observación de aves & 26 \\
\hline & & Visita a Centros biológicos & 6 \\
\hline \multirow{4}{*}{ De Aventura } & \multirow{4}{*}{20} & Caminatas, treeking & 38 \\
\hline & & Cabalgatas & 21 \\
\hline & & Paseo en bicicleta & 15 \\
\hline & & Escalada & 7 \\
\hline \multirow{3}{*}{ Cultural } & \multirow{3}{*}{19} & Convivencia con familias locales & 40 \\
\hline & & Participación en fiestas locales & 37 \\
\hline & & Participación en juegos locales & 23 \\
\hline \multirow{5}{*}{ Agroturismo } & \multirow{5}{*}{17} & Participación en actividades agrícolas & 42 \\
\hline & & En procesos artesanales & 25 \\
\hline & & En procesos agroindustriales & 12 \\
\hline & & En actividades pecuarias & 10 \\
\hline & & En pesca & 7 \\
\hline \multirow{3}{*}{ Místico } & \multirow{3}{*}{11} & Visita a lugares místicos & 34 \\
\hline & & Participar en ritual shamánico & 32 \\
\hline & & Participar en curaciones y limpias & 31 \\
\hline \multirow{3}{*}{ Histórico } & \multirow{3}{*}{10} & Visita a museos & 38 \\
\hline & & Visita a sitios históricos & 31 \\
\hline & & Visita a ruinas arqueológicas & 23 \\
\hline
\end{tabular}

\section{Promoción y venta del Producto Turístico Comunitario}

Esta se realiza utilizando diferentes medios, sobresalen página Web 21\%, 17\% lo hacen con folletos, $14 \%$ por redes sociales, $13 \%$ mediante videos y $12 \%$ por afiches; en menor proporción difunden a través de la televisión, radio y prensa escrita, estas son las razones para que los visitantes no se conformen con una excursión de horas hacia esos destinos, y optan por un mayor tiempo de permanencia en los CTC, un 32\% se quedan 1 día, un importante $56 \%$ se quedan entre 2 y 3 días y el 23\% dicen quedarse más de 3 días, especialmente en los feriados. El modo preferente de viajes de 
los turistas a las comunidades es en pareja y entre 3 y 5 personas el $48 \%$, entre 6 y 8 personas $38 \%$ y, el 14\% visitan solos.

\section{Conclusiones.}

Entre los beneficios del Turismo Comunitario (CTC) está la generación de empleos permanentes y temporales, evidencia la equidad de género y tienen acceso a servicios básicos como energía eléctrica.

Existen debilidades en abastecimiento de agua potable, $54 \%$ se abastecen con agua entubada, el manejo de desechos cuenta con pocos programas de tratamiento como reciclaje que son responsabilidades de los GADs.

Las comunidades y CTC gestionan al menos 6 tipos de turismo, ofrecen una gama de actividades en un medio natural, aprovechan su riqueza cultural y se benefician con la venta directa de la diversidad de productos locales que ofrecen, actividad alternativa que contribuye a elevar su calidad de vida comunitaria y eleva su autoestima.

En servicio de hospedaje, en la Región Andina, se ofrecen opciones de alojamiento en casas de familia, especialmente donde los visitantes tienen la oportunidad de convivir e interactuar con la comunidad anfitriona; mientras que en la Amazonia se ha desarrollado más el alojamiento en cabañas y también están las áreas destinadas para acampar, ya que, un importante número de visitantes tienen espíritu aventurero y optan por acampar. El costo por este servicio oscila entre $10 \mathrm{y}$ 30 dólares por noche que incluye cena y desayuno, es muy importante el fortalecer estos servicios, puesto que, los viajes hacia los sectores rurales requieren generalmente más de dos días de visita. 
Las 3/4 partes de los emprendimientos están asociados a la Federación Plurinacional de Turismo Comunitario del Ecuador (FEPTCE) y/o a una Red de Turismo Comunitario.

\section{Bibliografía.}

Burns, P., \& Holden, A. (1995). Tourism: A New Perspective. London: Prentice Hall.

Deller, S. (2010). Rural poverty, tourism and spatial heterogeneity. Annals of Tourism Research, $37(1), 180-205$.

Duffy, R. (2008). Neoliberalising Nature: Global Networks and Ecotourism Development in Madagascar. Journal of Sustainable Tourism, 16(3), 327-344.

Duterme, B. (2006). Expansion du tourisme international: gegnants et perdants. Alternatives Sud, 13(3), 7-22.

Feeroz, M. (2009). Effects of Environmental Degradation on Food Security in the St. Martin's Island of Bangladesh. Bangladés: National Food Policy Capacity Strengthening Programme.

Gascon, J., \& Cañada, E. (2005). Viajar a todo tren: Turismo, desarrollo y sostenibilidad. Barcelona: Icaria.

Grab, S., \& Linde, J. (2008). Regional Contrasts in Mountain Tourism Development in the Drakensberg, South Africa. Mountain Research and Development, 28(1), 65-71.

Hamilton, C. (2006). El fetiche del crecimiento. Pamplona: Laetoli.

Hampton, M. (2003). Entry points for local tourism in developing countries: evidence from Yogyakarta, Indonesia. Geografiska Annaler, 85(2), 85-101.

Henrici, J. (2007). Género, turismo y exportación: ¿llamando a la plata en el Perú? Anthropologica, 25(25), 83-101.

Hickman, L. (2007). The final call: In search of the true cost of our holidays. London: Eden Projects Books \& GBM.

Hutchins, F. (2007). Footprints in the Forest: Ecotourism and Altered Meanings in Ecuador's Upper Amazon. Journal of Latin American and Caribbean Anthropology, 12(1), 75-103.

Martínez, L. (2003). Los nuevos modelos de intervención sobre la sociedad rural: de la sostenibilidad al capital social. En B. Víctor, \& F. García (Edits.), Estado, etnicidad y movimientos sociales en América Latina. Ecuador en crisis (págs. 129-157). Barcelona: Icaria.

Mowforth, M., \& Munt, I. (1998). Tourism and Sustainability: New Tourism in the Third World. New York: Routlledge. 
Mowforth, M., Charlton, C., \& Munt, I. (2008). Tourism and Responsability: Perspectives from Latin America and the Caribbean. New York: Routdlege.

North, L., \& Cameron, J. (Edits.). (2003). Rural Progress, Rural Decay: Neoliberal Adjustment Policies and Local Initiatives. Bloomfield: Kumarian Press.

Nowicka, P. (2008). Vacaciones en el paraíso: turismo y desarrollo. Barcelona: Intermon OXFAM.

OMT. (2004). Turismo y atenuación de la pobreza: recomendaciones para la acción. Madrid: Organizacion Mundial del Trabajo.

Paniagua, A. (2002). Urban-rural migration, tourism entrepreneurs and rural restructurin in Spain. Tourism Geographies, 4(4), 349-371.

Prieto, M. (2008). Rosa Lema y la misión cultural ecuatoriana indígena a Estados Unidos: turismo, artesanías y desarrollo. En C. De la Torre , \& M. Salgado (Edits.), Galo Plaza y su época (págs. 157-191). Quito: FLACSO, Sede Ecuador.

Roux, F. (2013). Turismo Comunitario ecuatoriano, conservación ambiental y defensa de los territorios. Quito: Federación Plurinacional de Turismo Comunitario del Ecuador (FEPTCE).

Ruiz-Ballesteros, E. (2011). Social-ecological resilience and community-based tourism: An approach from Agua Blanca, Ecuador. Tourism Management, 32(3), 655-666.

Sasha Davis, J., \& Morais, D. (2004). Factions and Enclaves: Small Towns and Socially Unsustainable Tourism Development. Journal of Travel Research, 43(1), 3-10. 